\title{
Asentamientos informales y hábitat: un análisis de casos en la ciudad de Posadas, Argentina
}

\author{
Informal settlements and habitat: an analysis of cases in the city of Posadas, Argentina
}

Assentamentos informais e habitat: uma análise de caso na cidade de Posadas, Argentina

Walter F. Brites.

PhD. en Antropología Social. MSc. en Políticas Sociales. Investigador. Instituto de Estudios Sociales y Humanos. Consejo Nacional de Investigaciones Científicas y Técnicas. (IESyH-CONICET). Posadas, Argentina. briteswalter@yahoo.com.ar

(iD) http://orcid.org/0000-0003-2801-5792

Miguel, A. Avalos.

Lic. Antropología Social. Becario Doctoral CONICET. Consejo Nacional de Investigaciones Científicas y Técnicas. (IESyH-CONICET). Argentina. miguelavalos.012@gmail.com

(iD) http://orcid.org/0000-0001-9395-2032

Recibido: febrero 24 de 2020 Aceptado: abril 8 de 2020 Publicado: abril 13 de 2020

\section{RESUMEN}

Este artículo desarrolla un abordaje exploratorio, con análisis descriptivo, sobre distintos casos de asentamientos informales de la ciudad de Posadas, Argentina. Una ciudad en donde la emergencia de nuevos asentamientos y la cantidad de población que residen en ellos la convierte en un caso empírico, crucial para estos estudios. Se evidencian las vinculaciones entre hábitat, salud y pobreza, en el marco de los condicionantes socio-urbanos. Más allá de la vulnerabilidad en el acceso al suelo y al déficit de vivienda, los residentes de asentamientos atraviesan por problemas diversos en términos de empleo, alimentación, salud, educación, saneamiento ambiental, etc. Por otro lado, al margen de los constreñimientos estructurales, interesa conocer cómo experimentan las adversidades inherentes al entorno y cuáles son las prácticas individuales o colectivas que desarrollan para sortear las dificultades cotidianas.

Palabras clave: suelo urbano; servicios; vivienda; salud

\section{ABSTRACT}

This article develops an exploratory approach, with descriptive analysis, on different cases of informal settlements in the city of Posadas, Argentina. A city where the emergence of new settlements, and the amount of population residing in them, makes it an empirical case, crucial for these studies. The links between habitat, health and poverty are evident, within the framework of socio-urban conditions. Beyond vulnerability in access to land and housing deficit, residents of settlements face various problems in terms of employment, food, health, education, environmental 
sanitation, etc. On the other hand, apart from structural constraints, it is interesting to know how they experience the adversities inherent in the environment and what are the individual or collective practices they develop to overcome everyday difficulties.

Key words: urban land; services; living place; health

\section{RESUMO}

Este artigo desenvolve uma abordagem exploratória, com análise descritiva, de diferentes casos de assentamentos informais na cidade de Posadas, Argentina. Uma cidade onde o surgimento de novos assentamentos e a quantidade de população que neles habita o tornam um caso empírico, crucial para esses estudos. As ligações entre habitat, saúde e pobreza são evidentes, no quadro das condições socio-urbanas. Além da vulnerabilidade no acesso à terra e ao déficit habitacional, os moradores de assentamentos enfrentam vários problemas em termos de emprego, alimentação, saúde, educação, saneamento ambiental, etc. Por outro lado, além das restrições estruturais, é interessante saber como elas experimentam as adversidades inerentes ao meio ambiente e quais são as práticas individuais ou coletivas que desenvolvem para superar as dificuldades cotidianas.

Palavras-chave: terra urbana; serviços; habitação; saúde

\section{INTRODUCCIÓN}

De modo general, en la ciudad de Posadas, situada al norte de la Argentina, el fenómeno de la pobreza estructural está estrechamente relacionado con la problemática de la urbanización informal, expresada a través de la emergencia de los "asentamientos" o "toma de tierras". A diferencia de los grandes centros urbanos de la Argentina, Posadas es una ciudad de tamaño medio y fronteriza -alberga a 400.000 habitantes en su área conurbana-, $\mathrm{y}$, en su condición de capital de provincia, concentra la administración, servicios y actividades financieras.

Actualmente, la ciudad de Posadas está marcada por los efectos del proyecto hidroeléctrico Yacyretá (EBY), que implicó que la ciudad recibiera los embates de la elevación del río Paraná. Esta situación re-estructuró su forma urbana, generando cambios socioterritoriales, al igual que en los esquemas de ocupación del suelo urbano. Las obras de infraestructura, que materializó la Entidad Binacional Yacyretá, tales como la construcción de nuevos barrios, costanera, saneamiento de arroyos, reconexión de nuevas vialidades, entre otras de índole urbana, requirió la remoción y relocalización de muchos asentamientos hacia una nueva periferia, donde se erigieron grandes complejos habitacionales (Brites, 2014). No obstante, transcurrido el tiempo, han emergido otros asentamientos en distintas áreas de la nueva trama urbana y se han agregado a los asentamientos que permanecieron en los resquicios de las franjas de renovación urbana. Aunque es relevante señalar que los asentamientos más recientes se localizan en la periferia sur, condicionados por la disponibilidad de suelo excedente y por los vaivenes generales de la obra pública en el nuevo frente fluvial.

Hacia el año 2015, un relevamiento de la Municipalidad de Posadas, registró 63 asentamientos, con 5.302 familias (Municipalidad de Posadas, 2015). En tanto, para el año 2016, la Organización No Gubernamental Techo relevó en Posadas y su Distrito Capital 70 asentamientos informales que, de manera global, albergaban a 12.490 hogares (Techo, 2016). A pesar de la disparidad en los números, gran parte 
de los asentamientos censados presentan características compartidas como precariedad socio-habitacional, hacinamiento, acceso irregular a servicios de agua potable y energía, un casi inexistente trazado urbano al interior $y$, de manera generalizada, una irregularidad dominial en la propiedad del suelo, entre otros aspectos.

A pesar de las cuestiones generales de los asentamientos de Posadas, es necesario aclarar que la situación de muchos de ellos es compleja y heterogénea, por lo que es vital atender en su análisis varias dimensiones, entre ellas las referidas al poblamiento y tamaño de cada asentamiento, la evolución en el tiempo, su localización en el espacio urbano, las prácticas reivindicativas de sus residentes, las estructuras de oportunidades $y / o$ constreñimientos del contexto, la vinculación de la población con la legislación y las políticas urbanas, etc.

Con base en un estudio de casos, datos secundarios disponibles y entrevistas realizadas en campo, el artículo examina los problemas cotidianos que experimentan los residentes de tres asentamientos, indagando, como punto de partida: ¿Cuáles son las desventajas del entorno y del hábitat, y las repercusiones sobre calidad de vida de sus habitantes? Y, ¿cómo vivencian a diario las adversidades sanitarias y ambientales? Asimismo, ¿de qué modo la ubicación de los asentamientos, en el espacio urbano, configura modalidades diferenciales de vida para sus habitantes?

\section{METODOLOGÍA}

La metodología utilizada arrancó por un trabajo exploratorio y descriptivo, que combinó con instrumentos cualitativos de registro y análisis de la información, a partir de entrevistas semiestructuradas sobre calidad de vida, vivienda y hábitat, a residentes, referentes y líderes comunitarios. Básicamente, la investigación constituyó un análisis sobre un conjunto de asentamientos, tomados como casos testigos emblemáticos, localizados tanto en áreas periféricas como en zonas de reciente intervención y revalorización urbana, tales como el Viejo Rowing, Belén, y La Favela (Figura 1).

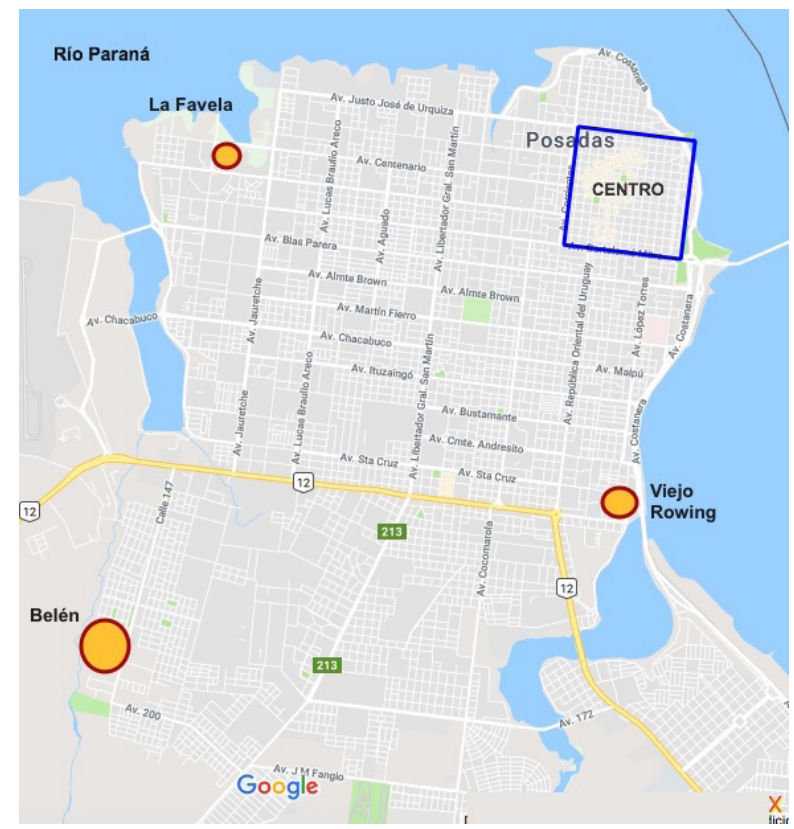

Figura 1. Ciudad de Posadas y ubicación de los asentamientos analizados

Fuente: Del autor, con base en Google Maps

De modo más general, los casos seleccionados para el estudio aquí presentado tienen características de asentamientos informales; fundamentalmente, por no poseer la certificación de propiedad de la tierra, ni permisos de ocupación, lo que deriva en situaciones de posesión denominadas "ilegales". Por otro lado, la pobreza, la precariedad habitacional y el acceso clandestino o irregular a los servicios de agua potable y energía eléctrica ${ }^{1}$, son otras tantas características compartidas.

Por otro lado, la perspectiva aquí propuesta, optó por no estructurar la investigación a partir del planteamiento de hipótesis a priori, como resulta usual en numerosas

1. Las prácticas vecinales del "enganche ilegal" al servicio de agua o de electricidad, y una derivación de una vivienda a otra, constituye una situación irregular para las empresas prestadoras de los servicios. 
investigaciones cualitativas (Taylor \& Bogdan, 1990). No obstante, con la finalidad de resolver problemas interpretativos y profundizar en nuevas dimensiones analíticas, la investigación estipuló estrategias de triangulación y complementariedad metodológica para el procesamiento y análisis de la información (Ander-Egg, 2004). En este sentido, para comprender la interacción de las múltiples dimensiones del fenómeno investigado, se recurrió a informes y datos de archivos existentes, como el Sistema de Información Geográfica SIG de la Municipalidad, Ong TECHO, Programa Promeba, C. Hábitat, Entidad Binacional Yacyretá, y la Cámara de Inmobiliaria de Misiones (CIM), entre otros documentos oficiales y no oficiales.

\section{RESULTADOS}

Asentamiento Viejo Rowing. Es un asentamiento de 35 años de antigüedad, el cual se ubica en las intersecciones de partes de las Chacras 26, 27, y de lo que quedó de la Chacra 222, afectada por el trazado de la costanera y acceso sur, en proximidad al sub-embalse del arroyo Zaimán (Figura 2.). El nombre Rowing encuentra su origen en el Viejo y desaparecido club de remo. Cabe recordar que gran parte del asentamiento ha sido relocalizado por la EBY, como consecuencia de las obras complementarias realizadas por Yacyretá en el área, cuando, hacia el año 2006, comenzaron las obras para elevar el frente fluvial más allá de la Cota 78. Actualmente, en el asentamiento radican unas 160 familias (820 personas aproximadamente).

En el relato de algunos vecinos, la ubicación del asentamiento, en una zona de intervención urbana y apertura de nuevos espacios públicos, les acarrea ciertas ventajas, no solo por la proximidad como espacio de ocio, sino que algunos lo aprovechan para el autoempleo en la venta ambulante (chipas, golosinas, agua refrescos, etc.). Otros residentes, en cambio, recuperan como valioso la cercanía al río Paraná, donde manifiestan que aún suelen pescar. Por otro lado, por la ubicación del asentamiento, hace que dispongan de trasporte urbano, comercios, escuelas y el hospital sea próximo.

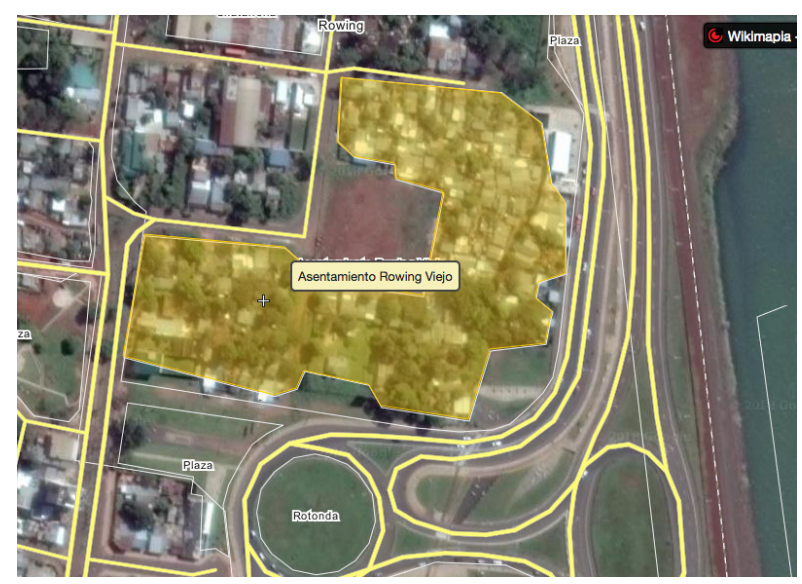

Figura 2. Mapa Asentamiento Viejo Rowing. Fuente: Wikimapia-Satélite 2018

Como situación adversa, las familias que hoy residen en el área tienen incertidumbre, por la aparición en el lugar de un supuesto dueño, propietario por mucho tiempo ausente, que reclama parte de las tierras, a lo que se agrega un malestar generalizado por rumores de su posible reubicación. Esta situación ha movilizado a los vecinos, quienes se organizaron para gestionar la mensura de los lotes que ocupan y gestionar una posible tramitación de certificados de propiedad (títulos), en función de los derechos que les corresponden por el tiempo de permanencia en el lugar.

Con base en una entrevista, una habitante manifestó que "la tenencia de los terrenos no está regularizada, $\mathrm{y}$, por eso, tenemos deficiencias de servicios; los vecinos estamos enganchado al agua y a la luz... Si nos solucionan este problema pagaríamos el agua, la luz y los impuestos".

De acuerdo con el testimonio de uno de los habitantes, se conoce que las familias del asentamiento atraviesan por gran cantidad de dificultades y preocupaciones. Así, muchas 
personas no pueden arreglar sus viviendas, debido a que sus ingresos económicos les permite solo proporcionarle el alimento o lo necesario para vivir, una situación que motiva a que la gente más pobre del barrio a veces reclame por su relocalización.

Con base en una entrevista, un residente señaló que hacia el interior del asentamiento las calles son horribles, y las aguas servidas corren libremente por las calles, lo que empeora los días de lluvias, cuando los pasillos se inundan $y$, en ocasiones, el agua ingresa en las casas y también en las letrinas. Por otro parte, el asentamiento no dispone de recolección de residuos, por lo que afloran mini-basurales en los perímetros del barrio, esperando que la municipalidad los retire. En reiteradas ocasiones, los vecinos han denunciado, ante los medios, las situaciones poco sanitarias con las que a diario conviven; y muchos residentes del asentamiento son conscientes de que se ven expuestos a diversas enfermedades. "Estamos expuestos a contaminación: viviendo así nos empeora la salud... Mi hija más chica y la hija del vecino tenían heridas por todos lados, y los doctores dijeron que es por el agua podrida de la canaleta" (entrevista a habitante de Viejo Rowing).

En la evaluación de las circunstancias que más preocupa a los residentes de este asentamiento, la cuestión sanitaria y socioambiental queda en un segundo plano, frente a la incertidumbre de la irregularidad en la que se encuentran y los temores por un posible desalojo, de no contar con un lugar donde vivir. Por otro lado, las mejoras urbanas en el entorno inmediato, el avance de nuevas edificaciones y la presión inmobiliaria, despierta a menudo una frustración para planificar a futuro la vida en el Viejo Rowing.

Chacra 159: La Favela. Denominado oficialmente como "asentamiento", por la Municipalidad de Posadas, La Favela se ubica en la zona oeste de la ciudad, a 7 kilómetros del centro urbano. Según el relevamiento de la ONG Techo, del año 2016, allí residen 250 familias (Techo, 2016). La Chacra 159, al igual que la colindante 158, conforman históricos "bolsones de pobreza" en la zona oeste de la ciudad (Díaz Orueta, et al., 2000), que comenzó a poblarse a inicios de la década de 1960, aunque encontró un fuerte impulso demográfico con la creación de los complejos habitacionales de Villa Cabello y Península Iprodha (Figura 3).

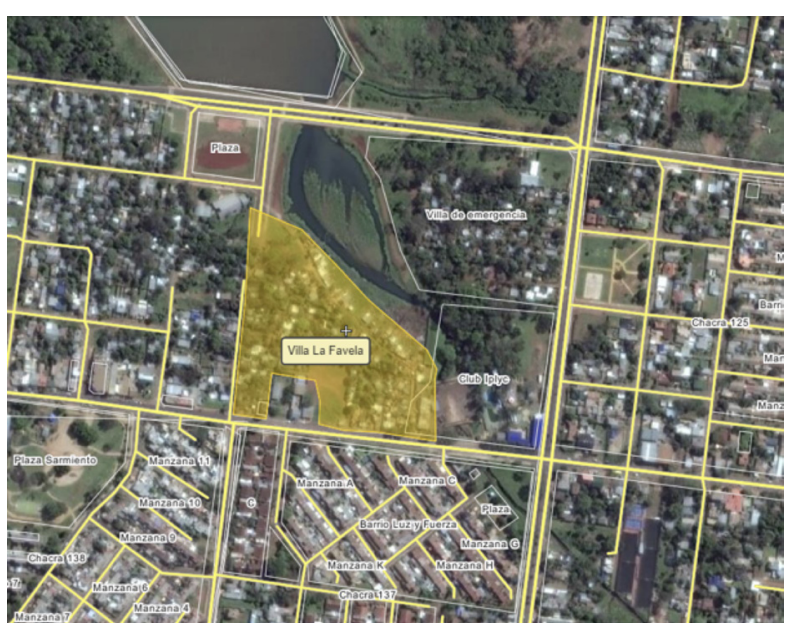

Figura 3. Asentamiento La Favela Fuente: Wikimapia-Satélite 2018

Las familias que se asentaron en la Chacra 159 provienen, fundamentalmente, de la Chacra 158, donde se produjo un crecimiento demográfico y el agotamiento de lotes disponibles, pero, también, La Favela recibió un flujo permanente de familias provenientes de otros asentamientos de la ciudad, de la zona centro-norte de la provincia y hasta de localidades cercanas del Departamento de Itapúa, Paraguay. Por otro lado, puede decirse que población que allí reside es relativamente joven, encontrándose una gran cantidad de niños y adolescentes.

El nivel de hacinamiento en el asentamiento es elevado. Las familias viven en casas precariamente instaladas, las cuales tienen reducidas dimensiones y muchas veces están construidas con residuos de pino y chapas de cartón. Mayormente, están ubicados en la costa del arroyo Antonica, sobre un terreno 
donde prima la roca basáltica, que contribuye a una escasa absorción del agua de lluvias y mayor escurrimiento, por lo que las viviendas están constantemente amenazadas por inundaciones.

Los riesgos ambientales, como las grandes lluvias 0 los fuertes vientos, generan adversidades para las precarias viviendas. Adversidades que, además, deben ser comprendidas e interpretadas de manera asociada con aquellos riesgos antropogénicos, resultado de las actividades generadas por la propia población, como la quema de basura a cielo abierto, aguas servidas ${ }^{2}$ y otros contaminantes.

La inexistencia de un servicio de recolección de residuos hacia el interior del asentamiento, hace que los desechos sean acumulados en mini-basurales ubicados en los intersticios de los pasillos internos o a los costados de algunas calles, y luego eliminados mediante incineración in situ. Por otro lado, la falta de aseo en espacios colectivos, a su vez, se configura en foco potencial para la propagación de enfermedades transmitidas por vectores, como el dengue, pero también favorece la proliferación de otros insectos y roedores, como alacranes, ratas, arañas o víboras.

En otras palabras, el riesgo ambiental y sanitario está siempre presente. Allí conviven, con elevados índices de humedad en el entorno, aguas servidas y la intermitente polución proveniente de la incineración de residuos sólidos domiciliarios. Esto provoca numerosos casos de enfermedades del sistema respiratorio, variadas patologías cutáneas y otras reacciones alérgicas. Considerando las manifestaciones de estas situaciones, es predecible que sus habitantes estén seriamente expuestos a diferenciales procesos de salud, enfermedad y atención (Báez, 2000).

2. Aguas residuales domésticas o aguas negras.
En cuanto a los procesos psicosociales, la violencia, sumada a la producción y comercialización de drogas ilícitas, es frecuentemente indicada por sus habitantes como causantes de problemas sociales y de salud, en especial de salud mental. Así, muchos residentes han manifestado problemas de convivencia, intranquilidad y quebranto al respecto.

El permanente temor y miedo en que viven muchas familias, constituye una emergente manera de sufrimiento social, que no suele encontrar solución por parte del Estado, aunque, a veces, reciben algún operativo asistencial que funciona como un paliativo que frecuentemente termina estigmatizando a la población que allí reside, y, otras veces, refuerza una lógica estatal punitivista, que no atiende la causal del problema. Allí, los jóvenes no cuentan con espacios de contención ni de esparcimiento; el espacio libre que solían usar como cancha de fútbol, por ejemplo, hoy es ocupado por viviendas y un templo evangelista.

Múltiples dimensiones de sus vidas los ubicarían en cierta parte de la "ciudad ilegal"; carecen de infraestructura y servicios básicos, hay ausencia de títulos de propiedad y autorizaciones de ocupación, a lo que se suma el desempleo, la falta de ingresos, los trabajos informales y mal remunerados. No obstante, sus cotidianidades se encuentran intrínsecamente ligadas a barrios aledaños de la "ciudad formal", como el complejo Villa Cabello y otras adyacencias de clase media. Las mujeres suelen trabajar como domésticas (Chacras 150, 138, 149). De manera similar, los hombres suelen emplearse como obreros de la construcción, albañiles, cortadores de pasto o cartoneros.

Por otro lado, una situación ventajosa del asentamiento, es que la población escolar, dispone de escuelas ubicadas en los complejos habitacionales colindantes (Escuela No. 7, 721, 730, BOP 8), y, además, cuentan con un centro de salud en la zona. De este modo, y a diferencia de los residentes 
en otros asentamientos, estos tienen accesos a ciertos recursos y servicios sumamente necesarios, sin los cuales la precariedad de sus condiciones de vida sería mayor.

Asentamiento Barrio Belén. Hacia el suroeste de Posadas, en el área de Itaembé Miní, se encuentra el barrio Belén. Su poblamiento se inició hacia el año 2000, mediante la toma progresiva y organizada de tierras, consolidando, en poco tiempo, un asentamiento informal. De acuerdo con el relevamiento de la ONG Techo (2016), el asentamiento alberga a 500 familias. El área en el que se localiza el barrio Belén es de exuberante vegetación y escasa densidad poblacional (Figura 4.), presentando un paisaje con características más rurales que urbanas. De hecho, en la zona hay granjas y varios clubes que aprovechan esta situación, como los clubes de Camioneros, de Suboficiales, de la Policía de Misiones, y Sarandí.

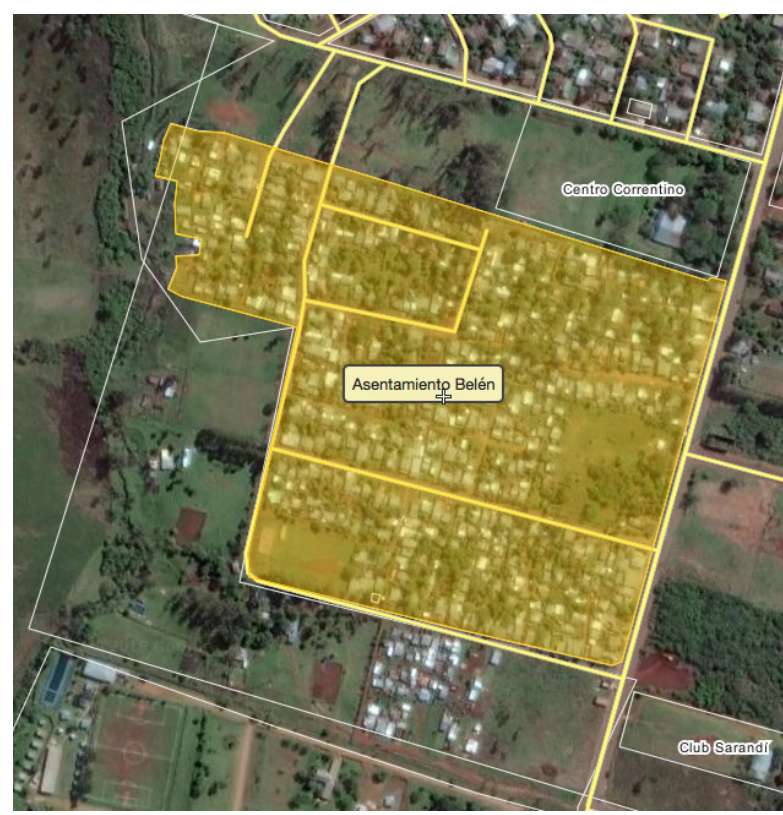

Figura 4. Mapa Asentamiento Belén Fuente: Wikimapia-Satélite 2018

Desde el inicio de su formación, la lejanía y la escasez de servicios han sido los pesares que más aquejan a la población del asentamiento. Su ubicación distanciada respecto a las áreas más urbanizadas de la ciudad, hace que la población dependa de un deficiente e intermitente servicio de transporte urbano para salir del barrio. Una situación que pone de relieve la vulnerabilidad socio-territorial, ya que, además, expresa los problemas de las distancias y de la marcada segregación social y espacial.

De acuerdo con una entrevista, un habitante señaló que es una "travesía ir y venir al centro de la ciudad" en alusión a los tiempos, las distancias y los costos del transporte. Aunque, por otro lado, es común escuchar, entre los residentes del asentamiento, que suelen hacer uso de los servicios disponibles en el cercano complejo habitacional Itaembé Miní, fundamentalmente de escuelas, centro de salud, comercios y minimercados, a los que se puede ir caminando o en bicicleta.

El barrio Belén se caracteriza por los altos niveles de pobreza de su población: familias con bajos ingresos y con necesidades básicas insatisfechas; por otro lado, la precariedad habitacional y de infraestructura en el barrio hace que el hábitat presente adversidades para la vida colectiva e individual. Hacia el interior del asentamiento no hay un definido trazado en cuadrículas; hay callejones sin salida y pasillos que generalmente solo son usados por sus propios habitantes.

Las casas están construidas mayoritariamente en madera, aunque, también, hay construcciones y anexos hechos en mampostería; además, muchas de ellas tienen problemas de hacinamiento y letrinas. Un informante recuerda que la mayor parte de las casas se han realizado progresivamente por mecanismo de autoconstrucción y ayuda mutua entre vecinos. Aunque también la ONG Techo ha construido viviendas, de manera frecuente, en el barrio.

El acceso al servicio de agua potable fue un problema sostenido en el asentamiento y los reclamos han sido indefinidos. La empresa de agua SAMSA, en acuerdo con 
la municipalidad, en un primer momento solo dispusieron de canillas públicas con baja presión. Por otra parte, la empresa de electricidad EMSA distribuyó un corto tendido eléctrico de baja potencia para las familias: "luz social" y sin alumbrado público. En el relato de un residente del barrio, allí la gente no puede acceder a servicios formales de conexión domiciliaria, porque no tienen títulos de las tierras, ni permisos de ocupación para solicitar el contrato de servicios que requieren las empresas. De modo general, las conexiones fueron realizadas por los propios habitantes $y$, en muchos casos, el tendido se realizó con base en una cadena de pases, de una familia a otra.

A pesar del acceso a la red de agua formal, muchas veces la extensión "informal o clandestina" genera cierto riesgo sanitario, debido a que las mangueras o caños utilizados son adosados precariamente, posibilitando filtraciones y, por lo tanto, carecen de seguridad para el consumo. La proximidad a basurales, áreas de quema, o arroyos contaminados, conllevan potenciales intoxicaciones digestivas e intestinales. Por otro lado, las viviendas asentadas en terrenos fangosos 0 próximos a estos y otros afluentes como las aguas negras, presentan más desventajas y vulnerabilidad por el anegamiento de suelo, una situación que se agrava en temporadas de lluvias.

En la historia del barrio podemos comprender cómo los vecinos se han socializado y organizado con base en los problemas y adversidades que debieron afrontar. En el proceso de conformación del asentamiento, los pobladores comenzaron a padecer carencias y necesidades ligadas al nuevo lugar de vida, compartieron las dificultades vecinales, buscaron interpretarlas y se socializaron para trabajar o reivindicar mejoras. En otras palabras, se trata de un proceso de construcción colectiva de demandas y reclamos, que derivó en la emergencia de nuevos actores colectivos y nuevas formas de acción social.
En la vida cotidiana, la socialización alrededor de los problemas comunes en el barrio Belén, motivaron incipientes y efímeras iniciativas de relacionamiento vecinal, tales como mítines, asambleas, colecta de firmas y petitorios para reclamar por servicios, como, por ejemplo, la instalación de un tanque de agua para reserva. Los procesos socio-organizativos, que comenzaron a materializarse, tuvieron como escenario un hábitat cargado de carencias, donde los residentes con el transcurrir del tiempo, fueron conformando asociaciones de carácter informal y territorial. Así, con base en una entrevista realizada, uno de los residentes manifestó: "fuimos sobre la marcha, organizándonos para resolver los problemas... después sí ya conformamos una comisión vecinal".

\section{Algunos Hallazgos}

Si bien los asentamientos informales de Posadas expresan una heterogeneidad de características y condiciones, en todos ellos existe, de manera extendida, una práctica común: sus habitantes son verdaderos productores de hábitat. En este sentido, apelando a los aportes de Flores Ortiz, entendemos a la producción social del hábitat (PSH) "todos aquellos procesos generadores de espacios habitables, componentes urbanos y viviendas, que se realizan bajo el control de auto-productores y otros agentes sociales que operan sin fines de lucro" (2012, p.73). Sin olvidar que son productores que trabajan bajo condiciones de adversidad, caracterizadas por pobreza, segregación, falta de recursos y múltiples situaciones de riesgos.

En el análisis de los asentamientos estudiados, podemos observar que, en el proceso de $\mathrm{PSH}$, se entrecruzan diversas prácticas, con diferentes alcances autogestionarios, desde la auto-producción familiar y progresiva de la vivienda, hasta variantes colectivas que implican cierto nivel organizativoy participativo a escala local. Además, de otros procesos, donde se crean espacios de deliberación pública entre vecinos, con el objetivo de 
transformar y mejorar el asentamiento como lugar de vida.

Con base en las vivencias cotidianas, las familias están frente a la posibilidad de interrelacionarse diariamente, generando prácticas autogestionarias y trabajos comunitarios, para dotar de bienes y servicios colectivos, como una forma extendida de producción de hábitat. Así, antes de conformarse una organización formal, los habitantes de los barrios crean asociaciones informales de corte inmediatista, para resolver los problemas más urgentes (Fernández,1992). Por ello, cuando los hogares de un mismo barrio están afectados por las mismas carencias, pueden, en su conjunto, intentar generar acciones colectivas/reivindicativas voluntarias como asociaciones barriales (Feijoo, 1984).

De modo general, las situaciones de adversidad y riesgos, a los cuales están expuestos los pobladores de asentamientos, están intrínsecamente relacionadas con las situaciones de hábitat inadecuado, así como a experiencias de segregación socio-urbana, siendo esta última un proceso que condiciona la distribución inequitativa en el territorio de satisfactores básicos para la calidad de vida de una población como: a. Oportunidades del entorno, en términos de acceso a la ciudad, al mercado de trabajo, b. Las condiciones del hábitat; esto es equipamientos comunitarios, infraestructura escolar, centros de salud, espacios de ocio, redes de servicios, etc., y c. Su relación con las políticas urbanas que establecen su legitimación, provisión de servicios, permanencia y/o remoción.

A pesar de que las familias residentes en asentamientos atraviesan por diferentes situaciones de precariedad, no obstante, desarrollan un estilo de vida adaptado a las características concretas de cada entorno en que viven. En este marco, se sostiene aquí, que la ubicación de cada asentamiento en el territorio urbano es una variable crucial al momento de analizar las adversidades $u$ oportunidades del entorno, las posibilidades y limitaciones de acceder a recursos y servicios, de sortear las restricciones y aprovechar las ventajas del medio.

De este modo, al pensar la cuestión de los asentamientos, es necesario re-problematizar diversas categorías como: lo público-privado, lo formal-informal, lo planificado/espontáneo, lo transitorio/permanente, lo legal/clandestino, considerando que los asentamientos de Posadas conjugan una heterogeneidad de situaciones de compleja y excepcional variabilidad, y que se expresan en una mezcla de características aquí enunciadas:

- Trazados urbanos regulares e irregulares combinados.

- Ocupaciones espontáneas u organizadas sobre tierras vacantes públicas y/o privadas.

- Diferenciales condiciones de pobreza de sus residentes.

- Acceso y tenencia no legal del suelo (pero con diferentes acuerdos y negociaciones).

- Autoproducción de viviendas y espacios colectivos, con y sin involucramiento de la legislación y las normativas urbanas.

- Localización en diversas áreas, tanto próximas a la centralidad urbana como en la periferia.

- Accesos a servicios, tanto por vía clandestina como legal provista por el Estado.

- Asentamientos chicos y grandes, nuevos y viejos, densificados y poco densificados.

- Viviendas, hábitat y servicios colectivos con diferentes evoluciones.

\section{CONCLUSIONES}

Desde una perspectiva exploratoria de análisis, la investigación ha demostrado las desventajas de orden estructural que implica el vivir en los asentamientos y los problemas conexos, referidos a la pobreza y las precarias condiciones de hábitat. Tomando los asentamientos estudiados, como casos testigos emblemáticos, se señaló que no todos son iguales, y se advierte que, a pesar de las desventajas estructurales condicionadas por la situación de pobreza, un hábitat inadecuado y deficitario, estas situaciones se redimensionan con los impactos de la segregación y la 
diferencial ubicación de cada asentamiento a lo largo del territorio urbano.

Un primer aspecto de la precariedad son las condiciones constructivas y deficitarias de las viviendas, a veces, con materiales extraído de la basura como láminas de cartón, reutilización de madera o chatarras. Un segundo aspecto, refiere a la carencia de servicios básicos para una óptima calidad de vida, espacios físicos degradados, suelos anegadizos y poco aptos para la urbanización, lo que acarrea diversas situaciones de riesgos y vulnerabilidades ambientales, así como sanitarias, con consecuencias para la salud de las familias.

Al margen del cúmulo de adversidades inherentes en cada asentamiento, las influencias del entorno urbano en el que se localizan intervienen como una estructura de recursos u oportunidades para la vida local de sus residentes. En síntesis, el análisis de los asentamientos de Posadas debe contemplar la importancia de su localización en el espacio de la ciudad.

En este sentido, hay asentamientos que se ubican en áreas periféricas, recluidas y segregadas socio-territorialmente, en espacios donde hay escasez de infraestructura, desarrollo de servicios y oportunidades. Por otro lado, están aquellos asentamientos que se hallan más integrados al espacio urbano, donde la revalorización urbana y la proximidad posibilitan mayor accesibilidad en áreas neurálgicas y actividades en la ciudad, dando lugar a una situación que no solo favorece, sino que amenaza, en orden a la permanencia debido a la especulación sobre las tierras, programas de intervención y cambios en la legislación/ordenamiento urbano.

Finalmente, la investigación puso de relieve, la importancia que el entorno juega, al configurar tanto adversidades o constricciones, así como estructuras de oportunidades en el devenir del barrio. Un contexto, donde, además, las experiencias de producción social del hábitat ponen de relieve diferentes situaciones de agencia, expresadas en prácticas socioorganizativas y locales, que implican el desarrollo de distintas lógicas de acción colectiva para solucionar los déficits del hábitat. Es decir, se trata de un conjunto de prácticas que permiten analizar cómo los residentes de estos asentamientos, no solo se desenvuelven bajo condiciones de adversidades, sino que desarrollan y dan forma a un conjunto de saberes y acciones colectivas orientadas a transformar el hábitat.

\section{REFERENCIAS}

Ander-Egg, E. (2004). Método y técnica de investigación social III. Buenos Aires. Ed. Lumen Humanitas.

Baéz, A. (2000). La salud materna en foco de riesgo. Editorial Universitaria. UNaM, Posadas.

Brites, W. (2014). Procesos socio-urbanos complejos. Hacia un abordaje multidimensional de la ciudad de Posadas, Argentina. En Millán M. y Brites, W. (Comp.). Ciudades Vivas. Imaginaciones sobre el territorio. Posadas. Creativa. 
Díaz Orueta, F., González Villar, C., Lourés, M. L., Sintes, L. (2000). Desarrollo urbano y pobreza: la ciudad de Posadas, Argentina. Universidad de Alicante.

Feijoó, M. (1984). Buscando un Techo. Familia y vivienda popular. Buenos Aires. CEDES.

Fernández, B. (2000). Lo colectivo de la sobrevivencia: entre la solidaridad informal y la debilidad formal. En: Cariola, C. (coord.) Sobrevivir en la pobreza: el fin de una ilusión. Caracas. Nueva Sociedad.

Florez Ortiz. E. (2012). Producción social de la vivienda y el hábitat Bases conceptuales y correlación con los procesos habitacionales. México. HIC.

Municipalidad de Posadas (2015). Diagnóstico del estado de situación de asentamientos en el municipio de Posadas 2014-2015. Coordinación del programa de regularización de espacios públicos y provisión de agua.

Taylor, S. y Bogdan, R. (1990). Introducción a los métodos cualitativos de investigación. Buenos Aires. Paidos.

TECHO. (2016). Relevamiento de asentamientos informales 2016. http://relevamiento.techo.org.ar/ index.html 